\title{
Development of Approaches to Creation of Active Vibration Control System in Problems of the Dynamics for Granular Media
}

\author{
Andrei P. Khomenko ${ }^{1}$, Sergey K. Kargapoltsev ${ }^{2}$ and Andrey V. Eliseev ${ }^{3, *}$ \\ ${ }^{1}$ Irkutsk State Transport University, 15 Chernishevskiy str., 664074, Irkutsk, Russia
}

\begin{abstract}
The article deals with the development of mathematical models and evaluation criteria of the vibration field in the dynamic interactions of the elements of the vibrational technological machines for the processes of vibrational strengthening of long-length parts with help of a steel balls working medium. The study forms a theoretical understanding of the modes of motions of material particles in interaction with a vibrating surface of the working body of the vibration machine. The generalized approach to the assessment of the dynamic quality of the work of vibrating machines in multiple modes of tossing, when the period of free flight of particles is a multiple of the period of the surface oscillations of the working body, is developed in the article. For the correction of vibration field of the working body, the characteristics of dynamic interactions of granular elements of the medium are taken into account using original sensors. The sensors that can detect different particularities of interaction of the granular medium elements at different points of the working body are proposed to evaluate the deviation from a homogeneous and one-dimensional mode of vibration field. Specially developed sensors are able to register interactions between a single granule, a system of granules in filamentous structures, and multipoint interactions of the elements in a close-spaced cylindrical structure. The system of regularization of the structure of vibration fields based on the introduction of motion translation devices is proposed using the multi-point sensor locations on the working body. The article refers to analytical approaches of the theory of vibration displacements. For the experimental data assessment, the methods of statistical analysis are applied. It is shown that the peculiar features of the motion of granular medium registered by the sensors can be used to build active control systems of field vibration.
\end{abstract}

\section{Introduction}

Modern technological machines work in intense dynamic modes of interaction of working bodies with the processed parts and working media [1-3]. Vibrating machines implement a variety of processes of transportation, classification and separation of granular mixtures, strengthening etc. [4-6]. Special aspects of joint motions of the elements of the system with unilateral constraints must be considered for the stable operation of these technological systems [7-8]. Fundamentals of the theory of vibrational technological processes and the vibratory displacements of the working media are reflected in the works [9-12]. This gives insight into the variety of motion forms and indicates the necessity of a comprehensive consideration of interactions between system elements in a spatial structure. Some of the issues of possible regular forms of working media are discussed in [8]. However, generalized approaches to integrated perception of the problems of interaction between the elements have not yet been given proper attention to the level of detail.

The aim of this work is to develop of a comprehensive approach to the forms of the trajectories of the medium elements in interaction with the vibrating support surface and methods for controlling integral parameters of dynamic state of the technical system.

\section{Formulation of a problem. Mathematical model of continuous tossing of material particles}

Generalized approach to the estimation of trajectories of particles with the formation of a free phase of flying followed by interaction with the vibrating surface is developed in the article. It is assumed that a horizontal surface makes the vertical harmonic vibrations. The interaction of material particles with the surface is inelastic in nature and is implemented taking into account the additional forces and viscous friction. The main interest is the continuous tossing of material particles. Analytical approach is developed to solve the problem of determining the trajectory of the working medium element. Concepts of a gap function, the conditions and order of separation from the surface are used in this approach.

\footnotetext{
* Corresponding author: eavsh@ya.ru
} 
The method of gap functions is the inverse problem relative to the direct problem of determination of a particle trajectory after detachment from the surface in a known point in time. The gap function allows carrying out detailed study of modes of tossing a material particle on the vibrating surface subject to unilateral constraints relations. In the case of harmonic oscillations of the surface, the gap function takes the form [8]:

$$
\begin{aligned}
& R_{H}\left(t, t_{0}\right)=A \sin \left(\omega t_{0}\right)-A \sin (\omega t)+ \\
& +A \omega\left(t-t_{0}\right) \cos \left(\omega t_{0}\right)-\frac{1}{2} g\left(t-t_{0}\right)^{2}, t \geq t_{0},
\end{aligned}
$$

where $A, \omega$ is the amplitude and frequency of oscillation of the surface, $t_{0}$ is the moment of separation. The gap function is based on the consideration of all possible trajectories. A set of possible trajectories in the phase of flight is shown in Figure 1.

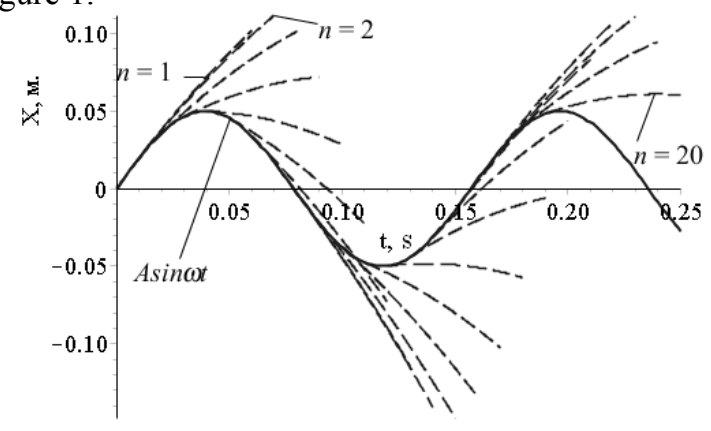

Fig. 1. A set of possible trajectories in the phase of flight

A number of sufficient conditions in terms of derivatives of the gap function are formulated to determine the moment of separation. The notion of the order of the separation for a multiple mode of tossing is used to differentiate different types of trajectories. In that case, trajectories with a multiple ratio of the approach time to the period of the surface oscillations are preferred in a certain way. A number of key characteristics of the trajectories are obtained using the gap functions for multiple modes of tossing. These characteristics are: the conditions of separation of material particles from the oscillation surface, the flight duration, the dependence on the constant additional forces and the forces of resistance from the working medium. In particular, the relationship between the trajectories of different multiplicity modes with the continuous tossing can be defined. A complex graph of the approach height of the material particles with different types of separation for a one-touch tossing mode is shown in Fig. 2. The curve $S$ is graph of height of throw of the particle from the area of separation of the third order depending on the frequency at a fixed oscillation amplitude $A=5 \mathrm{~mm}$; curves $k_{1}, \ldots k 5$ are the graphs of heights of throw of particles in case of the separation from the second-order separation area with multiplicity $1,2, \ldots 5$ accordingly. The variety of modes is determined by the detailed characteristics of the tossing modes of material particles. Therefore, these modes can be viewed as an effect of unilateral constraints when a material particle interacts with a vibrating surface. Concepts on the motions of the working media are based on concepts on the trajectory of the individual granules.

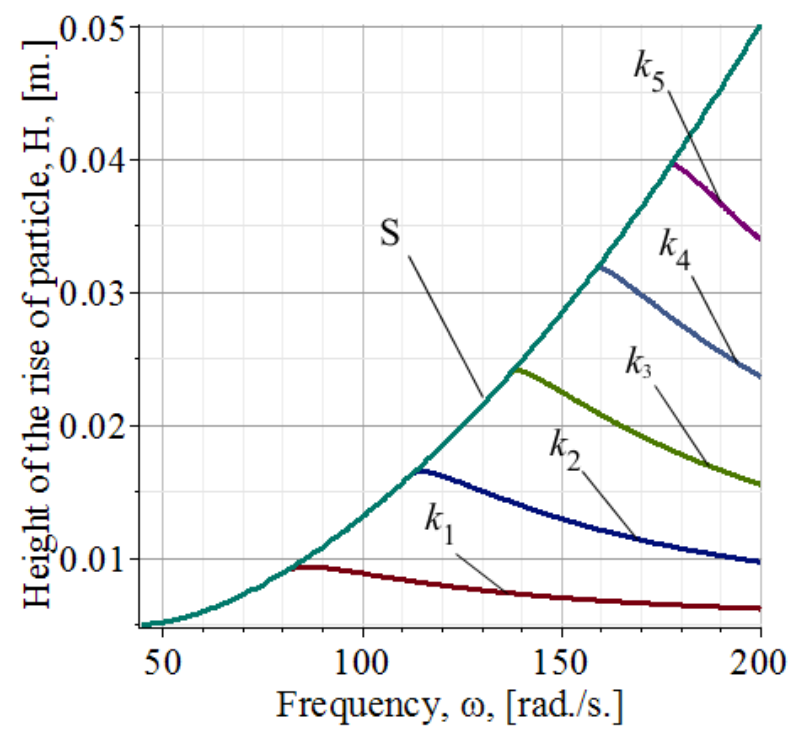

Fig. 2. The heights of throw. $S$ - is graph of height of throw of the particle from point separation of the third order. Curves $k_{1}, \ldots k_{5}$ - are the graphs of heights of throw of the particle from point separation of the second order

Thus, the dynamical interactions of the elements of technological complexes are studied due to effects of unilateral constraints. Analytical approaches are identified and developed during the evaluation of vibration technology processes with continuous tossing of the working medium. The generalized approach to problems of dynamic synthesis of vibration technologies during the continuous tossing is developed. The introduction of the concept of the gap function is the basis for the approach. Thus, the trajectories of motions of material particles are constructed with help of gap function in accordance with the properties of the interaction of the material particles with a vibrating surface. Mathematical models of formation of vibrational interactions are developed taking into account the factors having technological values, or criteria which include the conditions for the implementation of multiple modes, application of additional forces and elastic constraints [13-14].

\section{A mathematical model of vibration technology machines}

A mathematical model of the vibration stand which implements the process of vibration strengthening, with some assumptions, is reduced to a linear model with two degrees of freedom of small oscillations of a solid body placed on the elastic elements. The requirement of homogeneity and one-dimensionality of the vibrational field generated by one or more vibrators is a peculiar feature of the process. In the mathematical model, the influence of vibrators placed on the vibration table is reflected by the application of harmonic forces to specific points of the reference solid body. Adjustment 
of the vibration field is implemented by introducing a motion translation device with a predetermined rigidity and mass and inertia characteristics into the structure of the oscillating system. However, the vibrational field control system is also possible, when the mass and inertia elements are moved along the guides.

Solid body on elastic foundations under inertial excitation of the working body at a certain point (in this case, at the point of A) is shown in Fig. 3 in the form of a schematic diagram of the vibration machine. A model of the vibrating working body is formed by the solid body (1, Fig.3) of the mass $M$ with a moment of inertia $J$ in relation to the center of gravity $O$, elastic elements $k_{1}$, $k_{2}$ fixed at the points $A_{1}$ and $A_{2}$ at the distance $l_{1}$ and $l_{2}$ from the center of gravity $O$. It is assumed that the center of gravity is equal to the center of the swing for the solid body 1 . The harmonic force $Q_{l_{0}}=A_{f} \sin \left(\omega_{f} t\right)$ applied at the point $A_{l_{0}}$ at a distance $l_{0}$ from the point $O$ is a source of excitation. The device of motion transformation is inserted in the oscillation circuit to control the structure of vibration field. The device is characterized by stiffness $k_{0}$ and mass-inertia coefficient $L>0$. The device is installed vertically between the static surface 3 (Fig.3) and the center of gravity of the solid body 1 (Fig.3).

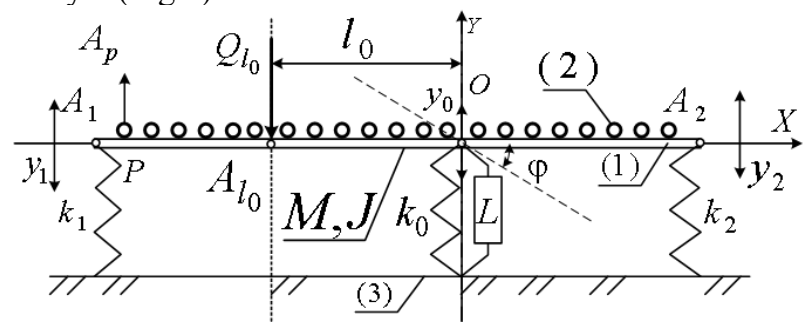

Fig.3. Design scheme of vibration technological machine. $y_{1}, y_{2}$ - the coordinates of the points $A_{1}, A_{2}, M$ - mass, $J$ - moment of inertia, $L$ - the device of motion transformation, $y_{0}$-coordinate of the center of mass, $\varphi$ - the angle of inclination

Configuring the vibration table in some cases is carried out by varying parameters of vibration table (weight of vibrators, stiffness of elastic elements, etc.) so that the amplitude and frequency of oscillations of the working body are in accordance with the technological requirements. In the present model problem, the technological requirements for the vibrating field points of the working body are homogeneity, frequency, amplitude and multiplicity of oscillations of the continuous tossing of the working media. In particular, the frequency for the fluctuation of the characteristic points $A_{1}, A_{2}$ of the working surface with equal amplitudes is given by:

$$
\omega_{0}^{2}=\frac{1}{M+L}\left(k_{0}+k_{1}+k_{2}+\frac{l_{2} k_{2}-l_{1} k_{1}}{l_{0}}\right) \text {. }
$$

The frequency range of vibrations in which the vibration table of the considered structure provides a homogeneous vibratory field of the working surface points, is extended if the "adjustment item" is included into the contour of the vibration table $[15,16]$.

\section{Approaches to the construction of the measuring system}

The formation of the experimental base of physical effects identified with help of a number of special sensors is implemented consequently with the development of the theoretical foundations of the technology of controlling the vibration field of the working body. Following the generalized principles, specialized sensors are developed that reflect the characteristics of the motion of the working mixture of the vibrational technological machines on the basis of indirect signs. Namely, the proposed measurement tools are based on the registering of dynamic effects of contact violation [17,18]. A variant of the schematic diagram of the sensor for evaluation the different dynamic states of the vibrating media is shown in Fig.4.

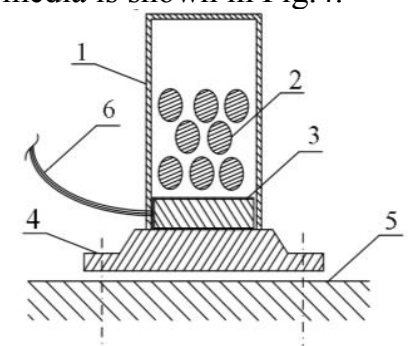

Fig. 4. A sensor for estimating the dynamic conditions of the working medium

Experimental sensor 5 is installed on the surface of vibration table and is comprised of a cylindrical body 1 ; inertial elements 2 of the working medium; a piezoelectric element 3; a fastening element 4; conductors of current 6 . The housing of the dynamic states evaluation sensor contains a few "representatives" of the working media of the vibration technological machine. It is assumed that resolution ability of sensor is sufficient for registration of the physical effects occurring inside the granular layer.

The assumption about the similarity or relationship between of the dynamic characteristics of the "working medium" of vibration technological machine and the "modeled medium" placed inside the sensor is the main design idea of the proposed sensors. Inertial elements of the "modeled medium" of sensor are equal to inertial elements of "working medium" of vibration technological machine. However, the problem of identity of dynamics properties of "modeled medium" and "working medium" has not been solved yet.

The method of four sensors to be simultaneously installed on the working body of the vibration table is proposed in the approach to control the vibration field by changing the mass and inertia characteristics of the working body.

The peculiar features of vibration of the working body as a solid body performing complex oscillatory motion at the harmonic excitation are taken into account by location of sensors. Method for analyzing the vibration field structure is focused on the measurement of the vertical component of the velocities, 
displacements and accelerations at the corresponding information carrier to determine the distribution of amplitudes of displacements, velocities and accelerations.

It is assumed that the homogeneity of the vertical motion of the working body of the vibration table is provided by the respective limiters of motion and by the ability to perform adjustments by keeping certain ratios of the parameters of motion in other directions except vertical.

Schematic diagram of the vibratory table in the form of the solid body undergoing vertical oscillations is shown in Fig.5. The working body of the vibration table 2 is mounted on the elastic elements 5 . Sensors 1 are installed at the corners of the working surface of vibratory table. Rails 4 with mass and inertia elements 3 that can be displaced by means of incremental principle of operation are mounted along the side surfaces of the working body of the vibratory machine.

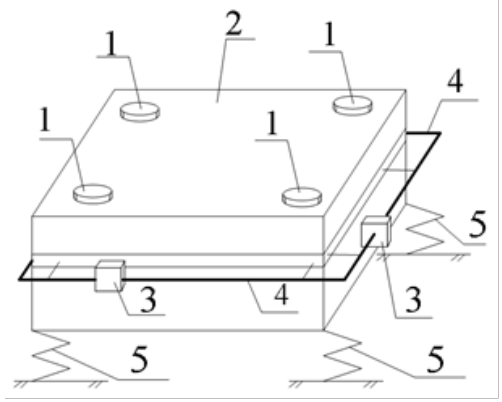

Fig. 5. Schematic diagram of the vibratory table in the form of one solid body which makes a vertical oscillation

The control capabilities are based on the assumption that there is a constructive position of the mass inertial elements, after the transition to which the vibrational field acquires the characteristics of homogeneity with four control points of the unmatched signals.

\section{Discussion of results}

Experimental testing efficiency of the proposed approach has been carried out in a production environment [19-20]. The performance of the sensors has been verified by special experiments in which statistical certainty of the signals is evaluated, and the dependences of forms of the vibrational modes on the "modeled medium" contained inside the casing of the sensors have been obtained.

A number of experiments on the measurement of signals with different number of "modeled medium" elements have been carried out to establish the dependence of the waveforms on the "modeled medium". The statistical characteristics of the signal, including the standard deviation, are determined by the number of elements of the "modeled medium" and by shape of the sensor casing. Peculiar features of functional dependencies between the basic parameters of the signal are of special interest. Graphics plots of the model sensor signals with 2 and 22 elements of a "modeled medium" which are represented by the vertical chains of steel balls inside the cylindrical casing are shown in Fig. 6 and Fig.7 respectively. The signals are represented by the process of "quasi pulsation" which is characterized by a periodic increase of the oscillation amplitude in the control points of a model vibratory table. The same processes are plotted by various graphs of signals from sensors by the same design, but with a different number of elements of "modeled medium".

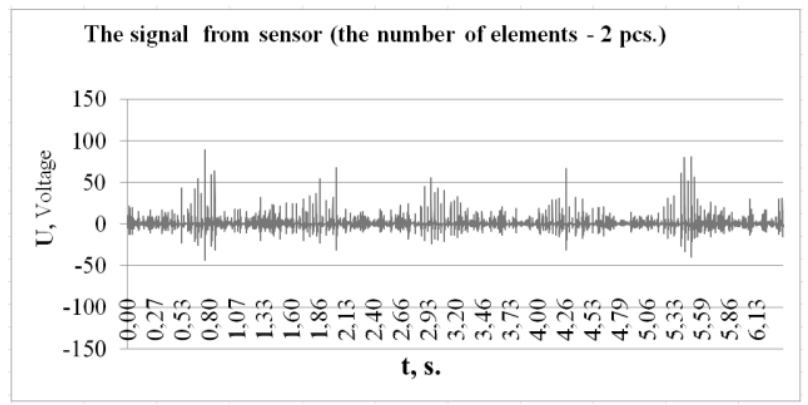

Fig. 6. The signal from sensor with 2 elements.

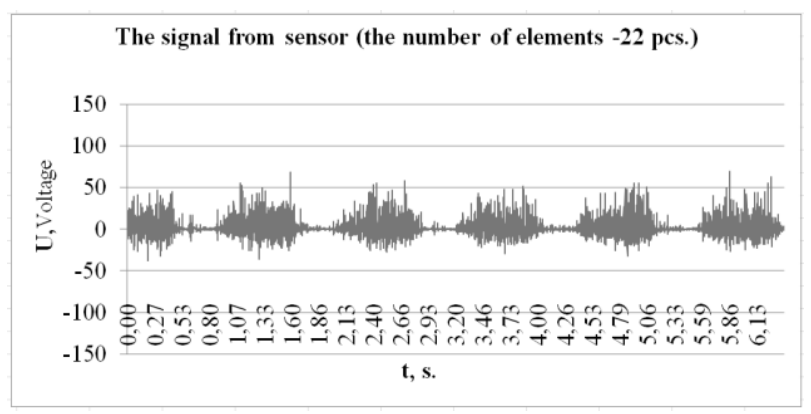

Fig. 7. The signal from sensor with 22 elements.

In addition, methods of mathematical statistics are used to justify the possibility of obtaining a technical result. Thus, the statistical significance of differences in signals from sensors which design includes the introduction of additional elements is checked on the basis of the homogeneity criterion. The registration of the useful signal is confirmed by the statistical significance of the differences between the signal I recorded by a sensor with the inertial elements and signal II recorded by a sensor without the inertial elements, on the basis of the Smirnov homogeneity criterion with a significance level of $\alpha=0.05$. Fig. 8 shows the empirical distribution functions of signals I and II.

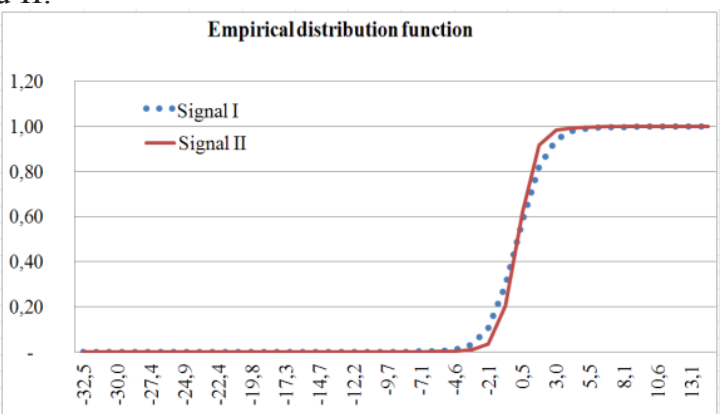

Fig. 8. Relative frequencies histogram for the signal I and signal II 
The hypothesis $\mathrm{H} 0$ is rejected ( $\mathrm{H} 0$ is a hypothesis of the homogeneity of the signal I and signal II) on the basis of the Smirnov (Kolmogorov-Smirnov) homogeneity criterion. The rejection of the homogeneity hypothesis indicates the statistical significance of differences between signal I and signal II. This is interpreted as the ability to obtain useful signal of the "modeled medium" of the sensor characterizing the dynamic qualities of the "working medium" of the vibration technological machine.

\section{Conclusion}

On the basis of studies related to the review and evaluation of measurement methods, it can be stated that fixation and control of current parameters of the vibration process - the displacement, velocity and acceleration - are the object of attention of the developers in the first place. However, for specific technical applications, such as vibration strengthening, compliance with a continuous tossing is of most interest. Despite the perfection of mathematical models, the transition from consideration of the interactions of one material particle with a vibrating surface to the concepts of the processes of interaction of the granular medium layer with the surface is quite complicated. Using sets of sensors to realistically reproduce the process of vibrational interaction can be considered as a solution. The controlling system of the vibration field is designed to configure the dynamic quality of the process of vibration strengthening of long-length parts using the working medium made of steel balls. It has been proposed to change the characteristics of the motion translation device (which imposes additional constraints) and mass and inertia characteristics of the working body by redistributing the locations of the inertial elements to control the vibration field. Measuring devices which construction is based on the assumption that the motion of the "working medium" is similar to that of the "modeled medium" elements of the sensor have been developed to evaluate the integral properties of the "working medium" motion for the vibration technological machine. The article presents an approach to the construction of the control system of the vibratory field of technological machines with the large working body formed by the solid body.

\section{References}

1. G.Ja. Panovko, Dynamics of vibrational technological processes (Moscow, Izhevsk: RHD Publ., 176 p., 2006) (In Russian)

2. Ju.R. Kopylov, Dynamics of vibro-impact hardening (Voronezh: Nauchnaja kniga Publ., 568 p., 2011) (In Russian)

3. A.P. Babichev, I.A. Babichev, Basics of vibration technology (Rostov-na-Donu: DGTU Publ., 620 p., 1999) (In Russian)

4. Ju.R. Kopylov, Spline estimation of the distribution parameters of surface layer quality in vibro-impact hardening. Journ. of Mech. Eng., No.7, pp. 53-60 (2016) (In Russian)

5. A.Ju. Marchenko, N.N. Kuznecova, G.V. Serga, New construction of equipment for surface hardening of parts in engineering production. Proceedings of the VII International scientificpractical conference (InMash-2015), pp. 95-99 (2015) (In Russian)

6. M.A. Tamarkin, D.V. Kazakov, A.S. Shvedova, R.V. Grebenkin, Improving the reliability of technological processes of machining by the dynamic methods of surface plastic deformation. Strength. Techn. and Coat., No.8 (128), pp. 23-27 (2015) (In Russian)

7. A.V. Eliseev, Features of mathematical modeling in problems of vibrating interactions between elements of technological systems subject to unilateral constraints relations. Proceedings of the DYVIS2015. XVIII International Symposium "Dynamics of vibroimpact (strongly nonlinear) systems". Moscow: IMASh RAN Publ., pp. 107-114 (2015) (In Russian)

8. A.V. Eliseev, V.V. Sel'vinsky, S.V. Eliseev, The dynamics of a vibratory interaction of elements of technological systems subject to unilateral constraints relations (Novosibirsk: Nauka Publ., 332 p., 2015) (In Russian)

9. I.I. Blehman, Vibrational mechanics (Moscow: Fizmatlit Publ., 400 p., 1994) (In Russian)

10. Clarence W. de Silva. Vibration. Fundamentals and Practice. Boca Raton, London, New York, Washington, D.C.: CRC Press, 2000. 957 p.

11. S. V. Eliseev, A. I. Artyunin, Applied theory of oscillations in problems of dynamics of linear mechanical systems (Novosibirsk: Nauka Publ., p. 459., 2016) (In Russian)

12. S.V. Eliseev, A.V. Lukyanov, Yu.N. Reznik, A.P. Khomenko, Dynamics of mechanical Systems with Additional Ties (Irkutsk: Irkutsk State University Publ., 315 p.)(In Russian).

13. S. V. Eliseev, K. K. Markov, Some problems of dynamics of the oscillatory process at not-holding ties. Mechanics and control processes (Irkutsk: Institute of Informatics Problems Publ., pp. 71-83., 1971) (In Russian)

14. V. I. Babitsky, Theory of vibro-impact systems (approximate methods)(Moscow:Nauka Publ., 1978, 352 p. (In Russian)

15. A.V. Eliseev, I.S. Sitov, D.X. Nguen, Some questions of formation of structure of vibrating fields of vibration table: features of the measurement system, configuration tools. Syst. Meth. Techn. (Bratsk: BrSTU Publ.), Bratsk, No.2 (30), pp. 17-26 (2016) (In Russian)

16. K. V. Frolov, Vibrations in engineering: Handbook in 6 volumes, Vol. 6. Protection of equipment from vibration (Moscow: Mechanical Engineering Publ. 1981, 452 p.) (In Russian)

17. S. V. Eliseev, A. P. Khomenko, Artyunin A. I., V. B. Kashuba, Sensor to monitor the dynamic state of vibration field (patent). The patent for useful model RF no. 166422 U1. Published: 27.11.2016, Bulletin No. 33 (2016) (In Russian) 
18. A.V. Eliseev, S.K. Kargapolcev, I.S. Sitov, A.V. Nikolaev, The integral estimation of the state parameters of granular medium under vibration interactions. 7th International Conference on Science and Technology. London, SCIEURO, 23-29 October 2016. pp. 53-68, (2016) (In Russian)

19. S.V. Eliseev, V.B. Kashuba, A.G. Pnjov, A.V. Eliseev, I.S. Sitov, Unilateral constraints in the dynamic interactions of the granular medium and the vibrating surface: the scientific and methodological substantiation of the technology of vibration hardening. Syst. Meth. Techn. (Bratsk: BrSTU Publ.), No.3 (23), Bratsk, pp. 17-31 (2014) (In Russian)

20. A.V. Eliseev, S.V. Eliseev, Approaches in the development of motion sensors in the problems of dynamics of granular media. Proceedings of Irkutsk State Technical University. No. 8 (115), pp. 20-35 (2016). DOI: 10.21285/1814-3520-2016-8-20-35. (In Russian) 